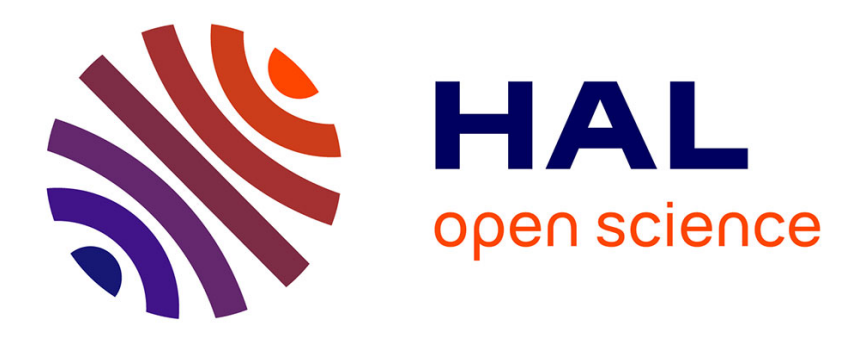

\title{
X-ray multilens interferometer based on Si refractive lenses
}

A. Snigirev, I. Snigireva, M. Lyubomirskiy, V. Kohn, V. Yunkin, S. Kuznetsov

\section{To cite this version:}

A. Snigirev, I. Snigireva, M. Lyubomirskiy, V. Kohn, V. Yunkin, et al.. X-ray multilens interferometer based on Si refractive lenses. Optics Express, 2014, 22 (21), pp.25842-25852. 10.1364/OE.22.025842 . hal-01572802

\section{HAL Id: hal-01572802 \\ https://hal.science/hal-01572802}

Submitted on 8 Aug 2017

HAL is a multi-disciplinary open access archive for the deposit and dissemination of scientific research documents, whether they are published or not. The documents may come from teaching and research institutions in France or abroad, or from public or private research centers.
L'archive ouverte pluridisciplinaire $\mathbf{H A L}$, est destinée au dépôt et à la diffusion de documents scientifiques de niveau recherche, publiés ou non, émanant des établissements d'enseignement et de recherche français ou étrangers, des laboratoires publics ou privés. 


\title{
X-ray multilens interferometer based on Si refractive lenses
}

\author{
A. Snigirev, ${ }^{1 *}$ I. Snigireva, ${ }^{1}$ M. Lyubomirskiy, ${ }^{1}$ V. Kohn, ${ }^{2}$ V. Yunkin, ${ }^{3}$ and S. Kuznetsov ${ }^{3}$

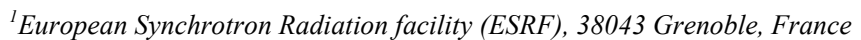 \\ ${ }^{2}$ National Research Center "Kurchatov Institute", 123182 Moscow, Russia \\ ${ }^{3}$ Institute of Microelectronics Technology RAS, 142432 Chernogolovka, Russia \\ snigirev@esrf.fr
}

\begin{abstract}
We report a multilens X-ray interferometer consisting of six parallel arrays of planar compound refractive lenses, each of which creates a diffraction limited beam under coherent illumination. Overlapping such coherent beams produces an interference pattern demonstrating substantially strong longitudinal functional dependence. The interference fringe pattern produced by multilens interferometer was described by Talbot imaging formalism. Theoretical analysis of the interference pattern formation was carried out and corresponding computer simulations were performed. The proposed multilens interferometer was experimentally tested at ID06 ESRF beamline in the X-ray energy range from 10 to 30 $\mathrm{keV}$. The experimentally recorded fractional Talbot images are in a good agreement with computer simulations.
\end{abstract}

(C)2014 Optical Society of America

OCIS codes: (030.0030) Coherence and statistical optics; (230.0230) Optical devices; (340.0340) X-ray optics.

\section{References and links}

1. A. Snigirev, V. Kohn, I. Snigireva, and B. Lengeler, "A compound refractive lens for focusing high-energy Xrays," Nature 384(6604), 49-51 (1996).

2. B. Lengeler, C. G. Schroer, M. Richwin, J. Tummler, M. Drakopoulos, A. Snigirev, and I. Snigireva, "A microscope for hard X-rays based on parabolic compound refractive lenses," Appl. Phys. Lett. 74(26), 3924 (1999).

3. B. Lengeler, C. Schroer, J. Tummler, B. Benner, M. Richwin, A. Snigirev, I. Snigireva, and M. Drakopoulos, "Imaging by parabolic refractive lenses in the hard X-ray range," J. Synchrotron Radiat. 6(6), 1153-1167 (1999).

4. V. Aristov, M. Grigoriev, S. Kuznetsov, L. Shabelnikov, V. Yunkin, T. Weitkamp, C. Rau, I. Snigireva, A. Snigirev, M. Hoffmann, and E. Voges, "X-ray refractive planar lens with minimized absorption," Appl. Phys. Lett. 77(24), 4058 (2000).

5. C. G. Schroer, O. Kurapova, J. Patommel, P. Boye, J. Feldkamp, B. Lengeler, M. Burghammer, C. Riekel, L. Vincze, A. van der Hart, and M. Kuchler, "Hard X-ray nanoprobe based on refractive X-ray lenses," Appl. Phys. Lett. 87(12), 124103 (2005).

6. A. Snigirev, I. Snigireva, M. Grigoriev, V. Yunkin, M. Di Michiel, S. Kuznetsov, and G. Vaughan, "Silicon planar lenses for high-energy x-ray nanofocusing," SPIE 6705, 670506 (2007).

7. I. Snigireva, A. Snigirev, V. Yunkin, M. Drakopoulos, M. Grigoriev, S. Kuznetsov, M. Chukalina, M. Hoffmann, D. Nuesse, and E. Voges, "X-ray Si-based Integrated Lens System for Wide Range of Hard Synchrotron Radiation,” AIP Conf. Proc. 705, 708-711 (2004).

8. A. Snigirev, I. Snigireva, G. B. M. Vaughan, J. Wright, M. Rossat, A. Bytchkov, and C. Curfs, "High energy Xray transfocator based on Al parabolic refractive lenses for focusing and collimation," JPCS 186, 012073 (2009).

9. G. B. M. Vaughan, J. P. Wright, A. Bytchkov, M. Rossat, H. Gleyzolle, I. Snigireva, and A. Snigirev, "X-ray transfocators: focusing devices based on compound refractive lenses," J. Synchrotron Radiat. 18(2), 125-133 (2011).

10. V. Kohn, I. Snigireva, and A. Snigirev, "Diffraction theory of imaging with X-ray compound refractive lens," Opt. Commun. 216(4-6), 247-260 (2003).

11. A. Schropp, R. Hoppe, J. Patommel, D. Samberg, F. Seiboth, S. Stephan, G. Wellenreuther, G. Falkenberg, and C. G. Schroer, "Hard $\mathrm{x}$-ray scanning microscopy with coherent radiation: Beyond the resolution of conventional x-ray microscopes," Appl. Phys. Lett. 100(25), 253112 (2012).

12. M. Drakopoulos, A. Snigirev, I. Snigireva, and J. Schilling, "X-ray high-resolution diffraction using refractive lenses," Appl. Phys. Lett. 86(1), 014102 (2005). 
13. A. V. Petukhov, J. H. J. Thijssen, D. C. T. Hart, A. Imhof, A. van Blaaderen, I. P. Dolbnya, A. Snigirev, A. Moussaid, and I. Snigireva, "Microradian X-ray diffraction in colloidal photonic crystals," J. Appl. Cryst. 39(2), 137-144 (2006).

14. A. Bosak, I. Snigireva, K. S. Napolskii, and A. Snigirev, "High-resolution transmission X-ray microscopy: a new tool for mesoscopic materials," Adv. Mater. 22(30), 3256-3259 (2010).

15. P. Ershov, S. Kuznetsov, I. Snigireva, V. Yunkin, A. Goikhman, and A. Snigirev, "Fourier crystal diffractometry based on refractive optics," J. Appl. Cryst. 46(5), 1475-1480 (2013).

16. A. Snigirev, I. Snigireva, V. Kohn, V. Yunkin, S. Kuznetsov, M. B. Grigoriev, T. Roth, G. Vaughan, and C. Detlefs, "X-Ray Nanointerferometer Based on Si Refractive Bilenses," Phys. Rev. Lett. 103(6), 064801 (2009).

17. H. F. Talbot, "Facts relating to optical science," Phil. Mag. Series 3 9, 401-407 (1836).

18. L. Rayleigh, "XXV. On copying diffraction-gratings, and on some phenomena connected therewith," Phil. Mag. Series 5 11(67), 196-205 (1881).

19. K. Patorski, "I The Self-Imaging Phenomenon and its Applications," in Progress in Optics, E. Wolf, ed. 1-108 (1989).

20. J. T. Winthrop and C. R. Worthington, "Theory of Fresnel Images. I. Plane Periodic Objects in Monochromatic Light," J. Opt. Soc. Am. 55(4), 373-380 (1965).

21. J. M. Cowley and A. F. Moodie, "Fourier Images IV: The Phase Grating," Pro. Ph. So. 76(3), 378-384 (1960).

22. M. V. Berry and S. Klein, "Integer, fractional and fractal Talbot effects," J. Mod. Opt. 43(10), 2139-2164 (1996).

23. Ya. M. Hartman and A. Snigirev, "Some examples of high energy X-rays phase contrast," in X-ray Microscopy IV (Bogorodskii Pechatnik, Chernogolovka, Moscow region, Russia), 429- 432 (1994).

24. A. Momose, S. Kawamoto, I. Koyama, Y. Hamaishi, K. Takai, and Y. Suzuki, "Demonstration of X-ray Talbot interferometry,” Jpn. J. Appl. Phys. 42(Part 2, No. 7B 7B), L866-L868 (2003).

25. T. Weitkamp, A. Diaz, C. David, F. Pfeiffer, M. Stampanoni, P. Cloetens, and E. Ziegler, "X-ray phase imaging with a grating interferometer,” Opt. Express 13(16), 6296-6304 (2005).

26. P. Cloetens, J. P. Guigay, C. De Martino, J. Baruchel, and M. Schlenker, "Fractional Talbot imaging of phase gratings with hard x rays," Opt. Lett. 22(14), 1059-1061 (1997).

27. A. A. Eliseev, N. A. Sapoletova, I. Snigireva, A. Snigirev, and K. S. Napolskii, "Electrochemical X-ray photolithography," Angew. Chem. Int. Ed. Engl. 51(46), 11602-11605 (2012).

28. A. Snigirev, I. Snigireva, V. Kohn, S. Kuznetsov, and I. Schelokov, "On the possibilities of x-ray phase contrast microimaging by coherent high-energy synchrotron radiation,” Rev. Sci. Instrum. 66(12), 5486-5492 (1995).

29. V. Kohn, I. Snigireva, and A. Snigirev, "Direct Measurement of Transverse Coherence Length of Hard X Rays from Interference Fringes," Phys. Rev. Lett. 85(13), 2745-2748 (2000).

30. C. G. Schroer, B. Lengeler, B. Benner, T. F. Günzler, M. Kuhlmann, A. S. Simionovici, S. Bohic, M. Drakopoulos, A. Snigirev, I. Snigireva, and W. Schröder, "Microbeam Production Using Compound Refractive Lenses: Beam Characterization and Applications,” SPIE 4499, 52-63 (2001).

\section{Introduction}

Since their development, the use of X-ray refractive lenses has rapidly expanded to the extent that they are now widely used on synchrotron beamlines [1-6]. Being in-line optics, the compound refractive lenses offer a number of advantages, they are easy to align, stable and relatively insensitive to misorientations and mechanical vibrations. The use of tunable systems such as transfocators with a variable number of lenses, offers focal length tunability that drastically extends the applicability of refractive optics [7-9]. They can be adapted to very high X-ray energies by modifying composition and number of lenses, and furthermore, refractive optics can be easily inserted and removed from the beam to allow fast switching of the beam size from the micrometer to nanometer scale.

The field of applications of refractive optics is not limited to beam conditioning, but can be extended into the area of Fourier optics, as well as coherent diffraction and imaging techniques $[10,11]$. Using the intrinsic property of the refractive lens as a Fourier transformer, the coherent diffraction microscopy and high resolution diffraction methods have been proposed to study 3-D structures of semiconductor crystals and mesoscopic materials [1215].

Another promising direction of refractive optics development is in-line X-ray interferometry. For example, a recently proposed bilens interferometer generated an interference field with a variable period ranging from tens of nanometres to tens of micrometers [16]. As an evolution of such systems, in this paper we propose a multilens interferometer in which more than two parallel lens arrays are arranged. The enlargement of 
the interferometer acceptance gives rise to the increases the contrast of the interference pattern, and a narrowing of the interference fringes.

The bilens system produces a steadily expanded sinusoidal interference field, while the multilens arrangement substantially changes the interference pattern by adding a strong longitudinal functional dependence. For simplicity we will describe the interference fringe pattern produced by the multilens interferometer using the Talbot imaging formalism [17]. It is known the object illuminated by a monochromatic plane wave can be self reproduced at Talbot distance $z_{T}=2 d^{2} / \lambda$, where $d$ is grating period and $\lambda$ is the wavelength of light. It was demonstrated that there is an infinite family of fractional Talbot images between the primary and secondary images [18-22]. The fractional Talbot distances are $z_{p q}=z_{T} p / q$, where $p$ and $q$ are integers, and $p<q$. However, the main set of distances is $z_{n}=z_{T} / 2 n$, where $n=1,2, \ldots$.

We emphasize that in the case of a multilens interferometer, the classical amplitude grid used in Talbot imaging is replaced by a collection of periodic line sources produced by linear lens arrays separated by the distance $d$. It is should be noted that the Talbot distance is measured from lens foci andthat these foci are reproduced in both the Talbot planes and fractional Talbot planes.

The Talbot imaging approach was already applied at hard X-rays in which a single Fresnel diffraction image of a grating was obtained with a microfocus X-ray generator in reflection geometry. Fractional Talbot imaging of the phase grating at reduced defocusing distances were used for coherence characterization and phase modulation of the object, as well as grating interferometer for phase contrast imaging [23-26]. Recently, Talbot imaging was also used for electrochemical lithography of Ni under coherent X-ray irradiation [27].

As a proof of concept we designed and manufactured "hexalens" composed of six lens arrays. The interference pattern formation was studied theoretically and computer simulations were performed. The optical properties of the interferometer were studied experimentally in the X-ray energy range $10-30 \mathrm{keV}$.

\section{Sixlens interferometer design and manufacturing}

The schematic diagram of hexalens interferometer image is shown in Fig. 1 (a). It consists of six arrays of identical, parallel planar compound refractive lenses (CRL) separated transversally by a distance $d$. Each compound refractive lens focuses the beam at the distance $z_{f}=F /\left(1-F / z_{0}\right)$, where $F=R / 2 N \delta$ is the lens focal length and $z_{0}$ is the source-to-lens distance, $R$ is the radius of curvature of one parabolic surface, $N$ is the number of double concave elements in the CRL, $\delta$ is the decrement of complex refraction index $n=1-\delta+i \beta$. Under coherent illumination each lens generates a coherent, diffraction limited focal spot of size $w_{f}=0.44 \lambda z_{f} / A_{\text {eff }}$, where $\lambda$ is the wavelength and $A_{\text {eff }}=0.66\left(\lambda z_{f} \delta / \beta\right)^{1 / 2}$ is the absorption limited effective aperture of the lens [1]. At a distance $z>3 z_{f} d / A_{\text {eff }}$ from the focal plane, the cones diverging from all six secondary sources overlap resulting interference in this region.

The hexalens interferometer was manufactured using a process involving electron beam lithography and deep etching into silicon $[4,6]$. The length and aperture of each double concave individual lens are 62 and $30 \mu \mathrm{m}$ respectively. Structures are $70 \mu \mathrm{m}$ deep. The radius of the parabola apex is $R=3.75 \mu \mathrm{m}$, and the minimum thickness between the parabola apexes is $2 \mu \mathrm{m}$. The split distance between lens arrays in the interferometer is $d=30 \mu \mathrm{m}$. A new interferometer design was applied in order to reduce split distance and to eliminate inrefracted $\mathrm{X}$-Rays which spoil the performance of the interference fringes at higher energies. 

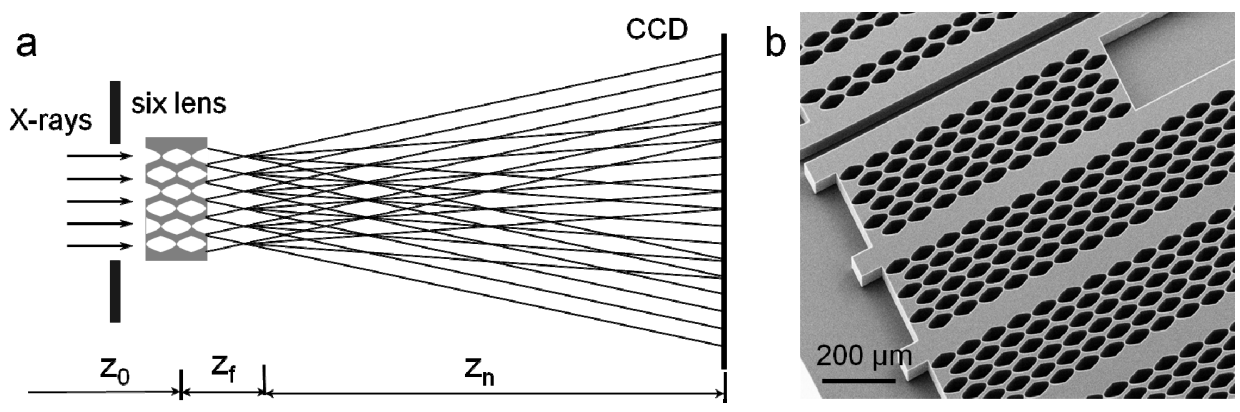

Fig. 1. (a) Schematic view of sixlens interferometer. (b) Scanning Electron Microscope image of the sixlens interferometers fabricated at the Si substrate.

The novelty of the design is that the lens arrays in the interferometer are arranged in a chessboard pattern; differently i.e. the arrays are shifted relative to each other (staggered) by the distance equal to half length of the single lens. An additional advantage of such lens arrangement is the coincidence of the lens array separation period and lens physical aperture. A Scanning Electron Microscope image of the six-lens interferometer is shown in Fig. 1(b). With the aim to simplify the use of interferometers in the experiments where energy tunability is required, we manufactured five sets of the hexalens on the same Si chip. All interferometer sets have a fixed focal distance $(F=4 \mathrm{~cm})$ for the chosen energy that was achieved by varying the numbers of individual lenses in each lens array. The chip covers a considerable $\mathrm{X}$-ray energy range from 10 to $50 \mathrm{keV}$. To choose the desirable working energy, one can switch from one interferometer set to another by a parallel displacement of the chip in the vertical direction. Table 1 summarized the main parameters of the hexalens sets.

Table 1. Parameters of sixlens interferometers

\begin{tabular}{|c|c|c|c|c|c|}
\hline $\begin{array}{c}\text { Set } \\
\text { number }\end{array}$ & $\begin{array}{c}\text { Energy, } \\
\text { keV }\end{array}$ & $\begin{array}{l}\text { Number } \\
\text { of lenses }\end{array}$ & $\begin{array}{c}\text { Lens length, } \\
\mu \mathrm{m}\end{array}$ & $\begin{array}{c}\text { Diffraction } \\
\text { Limited } \\
\text { Resolution, } \\
\text { nm }\end{array}$ & $\begin{array}{c}\text { Effective } \\
\text { aperture, } \mu \mathrm{m}\end{array}$ \\
\hline 1 & 10 & 10 & 618 & 190 & 13 \\
\hline 2 & 20 & 39 & 2416 & 87 & 17 \\
\hline 3 & 30 & 87 & 5392 & 63 & 21 \\
\hline 4 & 40 & 156 & 9670 & 55 & 23 \\
\hline 5 & 50 & 243 & 15064 & 53 & 23 \\
\hline
\end{tabular}

* Diffraction limited resolution calculated for single lens array in the interferometer

\section{Theory}

We have created a computer program to simulate the optical properties of X-ray multilens interferometers based on the general theory of X-ray phase contrast imaging [28]. The wave field of radiation from the point source at the distance $z_{0}$ is described by $\exp (i K z) P\left(x, z_{0}\right)$, where

$$
P(x, z)=\frac{1}{(i \lambda z)^{1 / 2}} \exp \left(i \pi \frac{x^{2}}{\lambda z}\right)
$$

is the Fresnel propagator which is a part of spherical wave in the paraxial approximation, $K=$ $2 \pi / \lambda$. Here $\lambda$ is the wavelength, $x$ is a transverse coordinate and $z$ is the coordinate along the optical axis. The exponential term $\exp (i K z)$ can be omitted because it does not influence the intensity.

Let us now consider the multilens interferometer, which consists of $M$ arrays of CRLs separated by distance $d$. The length of the interferometer is much smaller than the distance to 
the source. Therefore, to study its optical properties we treat the interferometer as a phase object which can be taken into account by the transmission function

$$
T(x)=\exp (-i K[\delta-i \beta] t(x)),
$$

where $t(x)$ is a variable thickness of the $M$-lens interferometer along the ray parallel to the optical axis. Here $t(x)$ is the sum of the thickness of each CRL

$$
t_{k}(x)=N\left(x-x_{k}\right)^{2} / R, \quad x_{k}=d\left(k-\frac{M+1}{2}\right), \quad k=1, \ldots M
$$

where $N$ is a number of individual lenses in CRL, $R$ is a curvature radius, $x_{k}$ is a position of the $k$-th lens center, $M$ is a number of CRLs in the interferometer.

A propagation of the wave through free space on the distance $z_{1}$ between the interferometer and the detector is described by a convolution of the wave function and $P(x$, $\left.z_{1}\right)$. The total expression can be transformed to a new form as a product $P\left(x, z_{t}\right)$ and a convolution of $T(x)$ and $P\left(x, z_{r}\right)$, where $z_{t}=z_{0}+z_{1}, z_{r}=z_{0} z_{1} / z_{t}$. From this, a relative intensity is calculated as follows

$$
I(x)=\mid a\left(\left.x_{0}\right|^{2}, \quad a\left(x_{0}\right)=\int d x_{1} P\left(x_{0}-x_{1}, z_{r}\right) T\left(x_{1}\right), \quad x_{0}=x z_{0} / z_{t}\right.
$$

We note that if $z_{0}$ is not large compared to $z_{1}$ then the image sizes is increased compared to the object, however, all features of the image stay the same.

The computer program based on these formulas allowed numerical simulation of the optical properties of the multilens interferometer for diverse parameters of the experimental setup. The finite size of the source was taken into account by a calculation of the convolution of the intensity for a point source and Gaussian with FWHM as $S\left(z_{1} / z_{0}\right)$ where $S$ is the source size.

Nevertheless, the properties of multilens interferometer can be formulated without calculations complex described above. It is of interest to analyze the main features of the interference fringes analytically. It is known that the $M$-lens interferometer transforms the incoming parallel beam to $M$ sets of divergent beams behind the focusing distance $z_{f}$. We will assume symmetrical case where $M$ is even. These focal lines are placed periodically in space along the axis $x$ with the period $d$. Our goal is to determine the distances $z_{n}$ where rays from all sources come to the optical axis $(x=0)$ with the same phase or with a difference which is integer number of $2 \pi$. It is clear that the relative intensity will be increased from 1 to $M^{2}$ at such distances. One can calculate the ray path $r_{k}$ from $k$-th CRL to the optical axis at the distance $z$ from the sources and consider a difference of ray paths for the arbitrary $k$ and $j$ sources. The result is

$$
r_{k}-r_{j}=r_{k j}^{(0)}=\frac{d^{2}}{z}\left[(k-j)\left(\frac{k+j}{2}-\frac{M+1}{2}\right)\right]
$$

Since $k, j, M$ are integer and $M$ is even the expression in the square brackets is also integer. Indeed, if $(k-j)$ is odd then $(k+j)$ is odd too and the expression in the second round bracket is integer. If $(k-j)$ is even then the expression in the round bracket is semi-integer but it is multiplied by even integer.

Thus the condition for constructive interference of the secondary sources produced by $M$ CRLs of the interferometer can be written as $d^{2} / z=\lambda n$ where $n$ is arbitrary integer number.

Distance $z_{T}=2 d^{2} / \lambda$ is a Talbot distance where the set of $M$ periodic line sources are reproduced. Halfway through the picture, a secondary Talbot image is formed which is shifted vertically from the primary image by half a period. At distances $z_{n}=d^{2} /(\lambda n)=z_{T} / 2 n$

\#216861 - \$15.00 USD Received 11 Jul 2014; revised 9 Sep 2014; accepted 10 Sep 2014; published 14 Oct 2014 (C) 2014 OSA 20 October 2014 | Vol. 22, No. 21 | DOI:10.1364/OE.22.025842 | OPTICS EXPRESS 25846 
between primary and secondary Talbot images the shrunken fractional Talbot images can be observed.

The next step is to calculate the period of the interference fringes. We repeat the calculations for all $x$ coordinates and obtain the result for the distances $z_{n}$ as follows

$$
r_{k}-r_{j}=r_{k j}^{(0)}-\frac{x d}{z_{n}}(k-j)=r_{k j}^{(0)}-\lambda n \frac{x}{d}(k-j)
$$

This expression shows that the period of interference fringes is $\Lambda=d / n$. The case where $M$ $=2$ is special because the expression in the square brackets of Eq. (5) is equal to zero, therefore the high contrast, sharp interference pattern exist at any distance. Correspondingly, the period depends directly on the distance and is equal to $\Lambda=\lambda z / d$.

Let us now estimate the width $w_{t}$ of the interference fringe maximum across the beam. We will assume that the distance $z$ is sufficiently large and all CRLs can interfere. In this case we can replace each secondary source by the real point source which is described by the function $P(x, z)$. Then, intensity can be written as $I(x, z)=(\lambda z)^{-1} K_{f}(x, z)$. Initially the function $K_{f}$ $(x, z)$ is a sum of $M$ and a double sum of cosines of complex argument which contains the coordinates of all CRLs. The double sum can be reordered to a more suitable expression which can be simplified significantly in the case of $z=z_{n}$.

We omit the calculations and present the result

$$
K_{f}\left(x, z_{n}\right)=M+2 \sum_{m=1}^{M-1}(M-m) \cos \left(2 \pi \frac{m}{\Lambda} x\right), \quad \Lambda=\frac{d}{n}
$$

We note that this expression is valid for the finite $M$ secondary sources produced by the interferometer, while the Talbot effect is applicable for the infinite periodic system. The period of the fringes does not depend on $M$; whereas the fringe intensity of the interference pattern is determined by $M$. For $x=0$ we have $K_{f}\left(0, z_{n}\right)=M^{2}$, at the same time a mean value of the fringe intensity averaged over the fringe period is equal to $M$.

To estimate a peak width we note that a numerical calculation of $K_{f}\left(x, z_{n}\right)$ shows that for large $M$ it is a peak which can be described approximately by a Gaussian: $K_{f}(x) \approx M^{2} \exp \left(-\alpha x^{2}\right)$. We can calculate the parameter $\alpha$ from the first term of the expansion of (7) in power series. As a result we have

$$
\alpha=\left(\frac{2 \pi}{\Lambda}\right)^{2} \frac{M^{2}-1}{12}, \quad w_{t}=\frac{1.665}{\alpha^{1 / 2}}=\Lambda \frac{0.918}{\left(M^{2}-1\right)^{1 / 2}}
$$

One can see that for the bilens interferometer of $M=2$ we have $w_{t}=0.52 \Lambda$ whereas $w_{t}=$ $0.92 \Lambda / M$ for large values of $M$.

To estimate a peak depth we can put $x=0$ and consider $z=z_{n}(1+s / n)$. Considering the general expression in this particular case, and making a replacement of this variable one can obtain

$$
K_{1 f}(0, s)=M+2 \sum_{m=1}^{M-1} \sum_{l=-(M-m-1) / 2}^{(M-m-1) / 2} \cos (2 \pi m l s)
$$

Now we apply the same procedure as above and expand the function over $s$. The first two terms of expansion are

$$
K_{1 f}(0, s)=M^{2}-(2 \pi s)^{2} C, \quad C=\sum_{m=1}^{M-1} m^{2} \sum_{l=-(M-m-1) / 2}^{(M-m-1) / 2} l^{2}=\frac{M^{2}\left(M^{2}-1\right)\left(M^{2}-4\right)}{720}(10)
$$

The calculation is cumbersome, but a physical meaning is evident because $C$ must be equal to zero for $M=0,1,2$.

\#216861 - \$15.00 USD Received 11 Jul 2014; revised 9 Sep 2014; accepted 10 Sep 2014; published 14 Oct 2014 (C) 2014 OSA 20 October 2014 | Vol. 22, No. $21 \mid$ DOI:10.1364/OE.22.025842 | OPTICS EXPRESS 25847 
Now it is easy to calculate $w_{l}$ as follows

$$
w_{l}=\frac{d^{2} w_{s}}{\lambda M^{2}} \approx \frac{7.111 \Lambda^{2}}{\lambda M^{2}} \approx 8.438 \frac{w_{t}^{2}}{\lambda},
$$

where $w_{s}$ is FWHM for the function (10). We note that the relation between $w_{l}$ and $w_{t}$ is very similar to the same relation for the focus spot and differs only by numerical coefficient 8.438 instead of 7.850. This is further evidence that the fractional Talbot effect is focusing.

\section{Experimental results}

The experimental tests of the sixlens interferometer were carried out at the Micro Optics Test Bench (MOTB) of the ID06 ESRF beamline. A liquid nitrogen cooled Si-111 double crystal, fixed exit monochromator was used to adjust the X-ray energy in the range from $10 \mathrm{keV}$ to 30 $\mathrm{keV}$. The Si sixlens system was mounted at the stage with all necessary rotation and translation movements at a distance $z_{0}=56 \mathrm{~m}$ from the source [see Fig. 1(a)]. Interference patterns were recorded with a high resolution x-ray CCD camera with a spatial resolution about $1.3 \mu \mathrm{m}(0.65 \mu \mathrm{m}$ pixel size). The typical exposure time was 2 seconds during a $7 / 8$ beam bunch mode ( $200 \mathrm{~mA}$ current).

As it was mentioned earlier, the foci of the six lens arrays in the interferometer form a periodic structure and therefore the foci are reproduced at the Talbot distances and are reproduced with a different scale in the fractional Talbot distances. Considering the parameters of hexalens it can be easily estimated that for $12 \mathrm{keV} \mathrm{X-rays,} \mathrm{the} \mathrm{Talbot} \mathrm{distance}$ $z_{T}$ is in the order of $18 \mathrm{~m}$. Taking into account the beamline characteristics (energy range, length) and CCD spatial resolution, the interference fringes were observed at the fractional Talbot distances $z_{n}$. Therefore the CCD was always placed at distance $z_{1}=z_{f}+z_{n}$. The quality of the fringes produced by a multilens system can be described quantitatively using the visibility $V=\left(I_{\max }-I_{\min }\right) /\left(I_{\max }+I_{\min }\right)$, where $I_{\max }$ and $I_{\min }$ are the irradiances corresponding to the maximum and nearby minimum in the fringe system, respectively.

To characterize the interferometer at $12 \mathrm{keV}$, the fractional $1 / 6$ Talbot distance which corresponds to $z_{n}=3 \mathrm{~m}$ was chosen. The observed interference pattern and intensity variation obtained for the line through the center of the fringe pattern are shown in Fig. 2. The measured fringe spacing was $\Lambda=10.3 \mu \mathrm{m}$, which is in very good agreement with the calculations: the measured FWHM of the fringe maximum is $3 \mu \mathrm{m}$, while according to the calculations it should be around of $1.7 \mu \mathrm{m}$. The broadening of the measured fringe width is because of the finite size of the source. The source size was measured by a B-fiber interferometry technique [29], which during the interferometer tests was in the order of 45 microns. The measured fringe visibility was approximates $73 \%$, while the theoretical contrast was around $88 \%$. Let us introduce the spatial coherence length described as $l_{\text {coh }}=\lambda z_{0} / S$, where $S$ is the source size. For the $45 \mu \mathrm{m}$ source size at $54 \mathrm{~m}$ from the source it is in the order of $130 \mu \mathrm{m}$. The width of the interferometer is $180 \mu \mathrm{m}$; therefore we cannot exclude the influence of the partially coherent illumination that reduces the contrast of the fringe pattern.

\#216861 - \$15.00 USD Received 11 Jul 2014; revised 9 Sep 2014; accepted 10 Sep 2014; published 14 Oct 2014

(C) 2014 OSA 20 October 2014 | Vol. 22, No. $21 \mid$ DOI:10.1364/OE.22.025842 | OPTICS EXPRESS 25848 

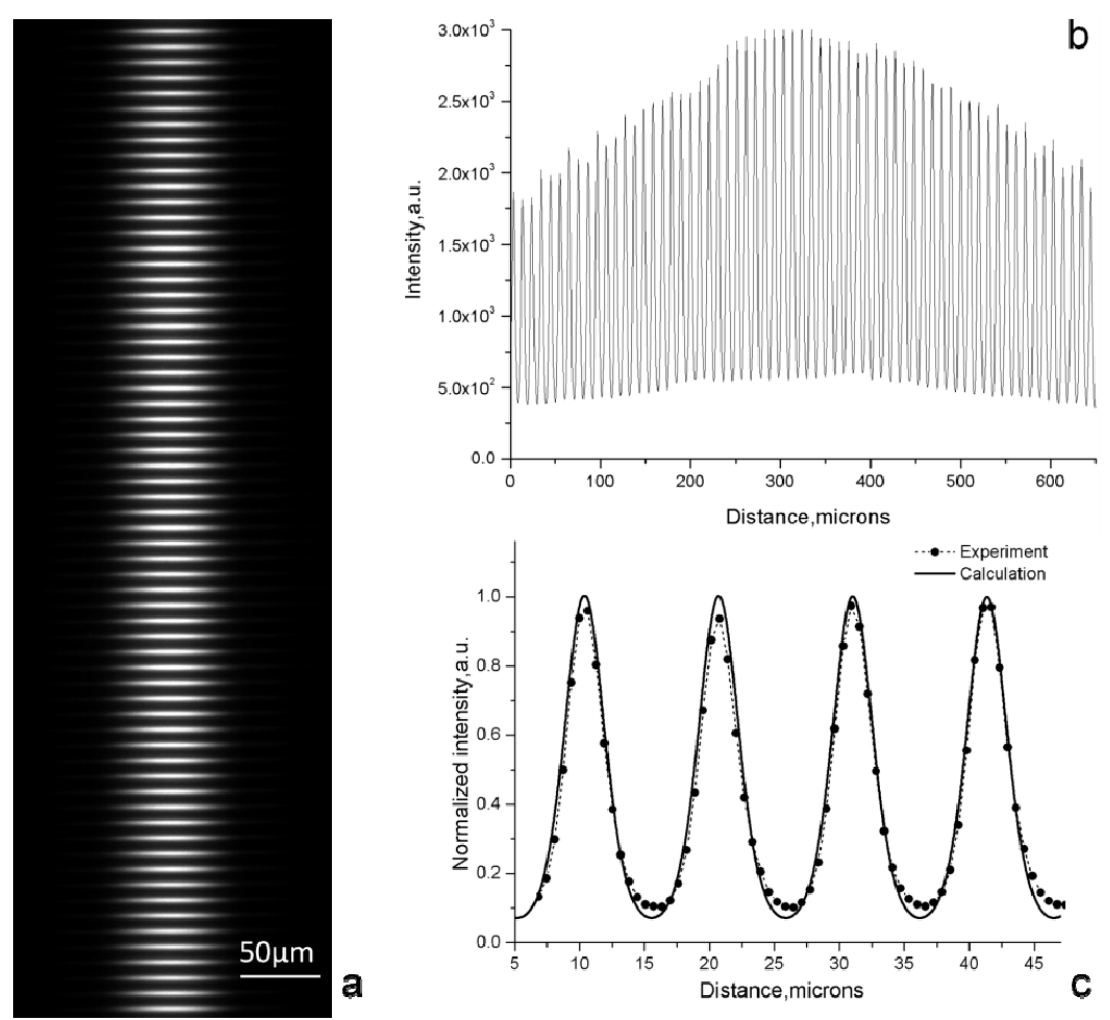

Fig. 2. (a) An interference pattern generated by a hexalens, recorded with $12 \mathrm{keV} \mathrm{X}$-rays. (b) The intensity variation obtained for the line though the centre of the fringe pattern. The contrast visibility is approximately $71 \%$. (c) The comparison of the intensity variations of the interference pattern experimentally measured and calculated one.

Let us estimate the stability of the interference pattern in terms of depth of field. Similar to the criteria for refractive lenses depth of focus [30], we define the interferometer depth of field as a range of distances along the optical axis trough which the lateral size of interference fringe (FWHM) is less than $\sqrt{ } 2$ of its value at the exact Talbot imaging distance. For this we placed the hexalens at the distance $z_{n}=3.06 \mathrm{~m}$ and then we scanned it along the optical axis in the interval $\pm 100 \mathrm{~mm}$ with $1 \mathrm{~mm}$ step around exact position. At each position the interference pattern was registered and the lateral size of the interference fringe was measured together with the contrast. Regarding the criteria expressed above a depth of field in the order of $80 \mathrm{~mm}$ was obtained which is in a very good agreement with calculations. It should be noted that within these depth of field the fringe contrast was reduced in the order of $20 \%$. For practical applicability of the interferometer the large depth of field is very important.

The sixlens interferometer was tested with $24 \mathrm{keV} \mathrm{X-rays} \mathrm{and} \mathrm{the} \mathrm{interference} \mathrm{pattern} \mathrm{was}$ registered at the fractional $1 / 12$ Talbot distance which is $z_{n}=3 \mathrm{~m}$. Experimentally measured fringe spacing of the recorded interference pattern was $6.3 \mu \mathrm{m}$ and FWHM of the fringe maximum was $2.7 \mu \mathrm{m}$. The measured contrast was around $40 \%$, which $30 \%$ lower than was expected from the calculations. We would like to note that the spatial coherence length at 24 $\mathrm{keV}$ is in the order of $70 \mu \mathrm{m}$. This means that only 3 lens arrays are illuminated coherently, which leads to the broadening of the interference fringes and decreasing of the fringe visibility. 

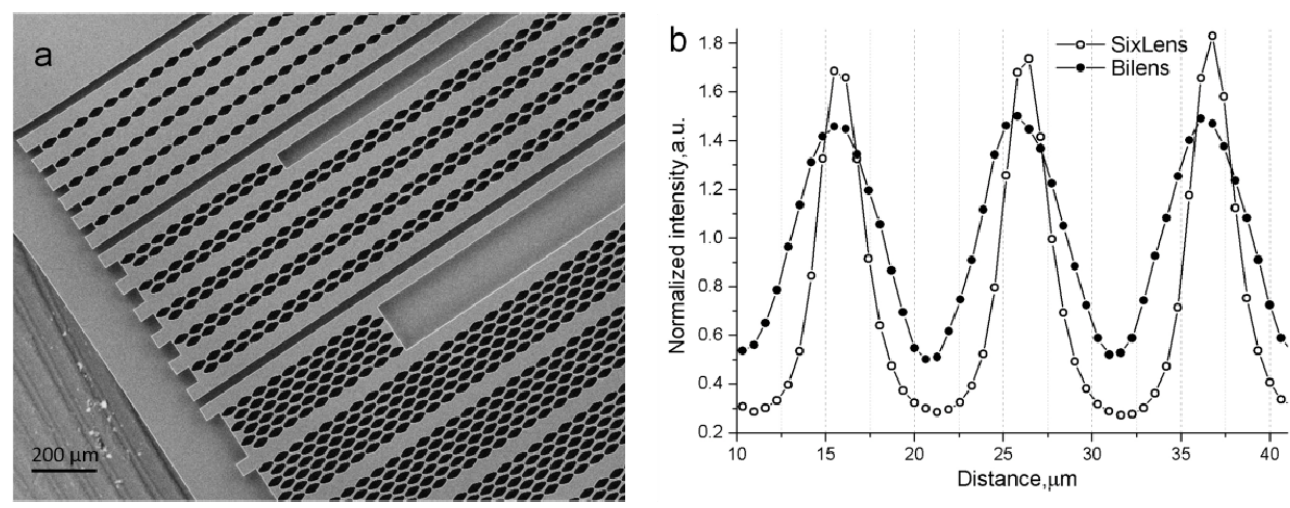

Fig. 3. (a) Scanning Electron Microscopy image of bilens and sixlens interferometers and (b) comparison of the fringe patterns produced by bilens and sixlens interferometers.

To demonstrate the narrowing of the interference fringe maximum in the case of the hexalens, the interference pattern produced by a bilens interferometer was recorded under identical experimental conditions: X-ray energy was $12 \mathrm{keV}$ and distance $z_{n}=3 \mathrm{~m}$. The bilens interferometer consists of two lens arrays with the separation period of $30 \mu \mathrm{m}$. The measured fringe spacing (peak-to-peak distance) of the bilens interference pattern was $\Lambda=10.3 \mu \mathrm{m}$. The comparison of the intensity variation through the center of the fringe pattern for the bilens and sixlens interferometers is shown in Fig. 3. The measured FWHM of fringe maxima for the bilens interferometer is $5.2 \mu \mathrm{m}$, as for the sixlens interferometer it is $3 \mu \mathrm{m}$. The results show a narrowing of the interference fringes but not as much as expected. As discussed above, the reason for this is a finite source size and partial coherent illumination. It is clearly seen that the contrast of the fringes produced by sixlens interferometer is superior to the fringe contrast of the bilens.

Hexalens was also experimentally tested with the point secondary source produced by $\mathrm{Si}$ planar refractive lens. The sketch of the experimental setup is shown in Fig. 4. The lens consisted of 26 individual lenses with radius of parabola apex of $6.25 \mu \mathrm{m}$ and aperture $50 \mu \mathrm{m}$. The Si lens was located at $z_{01}=55 \mathrm{~m}$ from the source. At energy $E=12 \mathrm{keV}$ it has a focal distance $z_{f l}=3.5 \mathrm{~cm}$. The interferometer was placed at the distance $z_{0}=550 \mathrm{~mm}$ from the secondary source. In the case where incident radiation comes from a point source at a finite distance $z_{0}$, the spherical wave approximation has to be considered. It is readily shown that the fractional Talbot image will be magnified by a factor $\left(z_{n}+z_{0}+z_{f}\right) / z_{0}$ and interference occurs at the distance $z_{\mathrm{n}}$ given by:

$$
\frac{1}{z_{f}+z_{n}}+\frac{1}{z_{0}}=\frac{1}{z_{f}^{(\infty)}+z_{n}^{\infty}}, \quad z_{f}^{\infty}=\frac{R}{2 \delta N}, \quad z_{n}^{\infty}=\frac{d^{2}}{\lambda n}, \quad z_{f}=\frac{z_{f}^{\infty}}{1-z_{f}^{\infty} / z_{0}}
$$

A high order fractional 1/40 Talbot image was registered at the distance $z_{20}=3.8 \mathrm{~m}$ from the lens array foci, with a magnification factor of around 7. The measured fringe spacing is $11.5 \mu \mathrm{m}$ and FWHM of the interference fringe maximum is $3.1 \mu \mathrm{m}$ [Figs. 4(b) and 4(c)]. The measured visibility of the interference pattern is $91.5 \%$, which corresponds to the secondary source size in the order of $220 \mathrm{~nm}$. It should be mentioned that for the plane wave illumination, the 1/40 fractional Talbot image can be registered at $45 \mathrm{~cm}$ distance from the lens focal lines. The period of the fringe pattern is $1.5 \mu \mathrm{m}$ and the FWHM of the fringe maximum is $0.25 \mu \mathrm{m}$ which beyond the resolution of current CCD with scintillation screen based X-Ray detectors. 

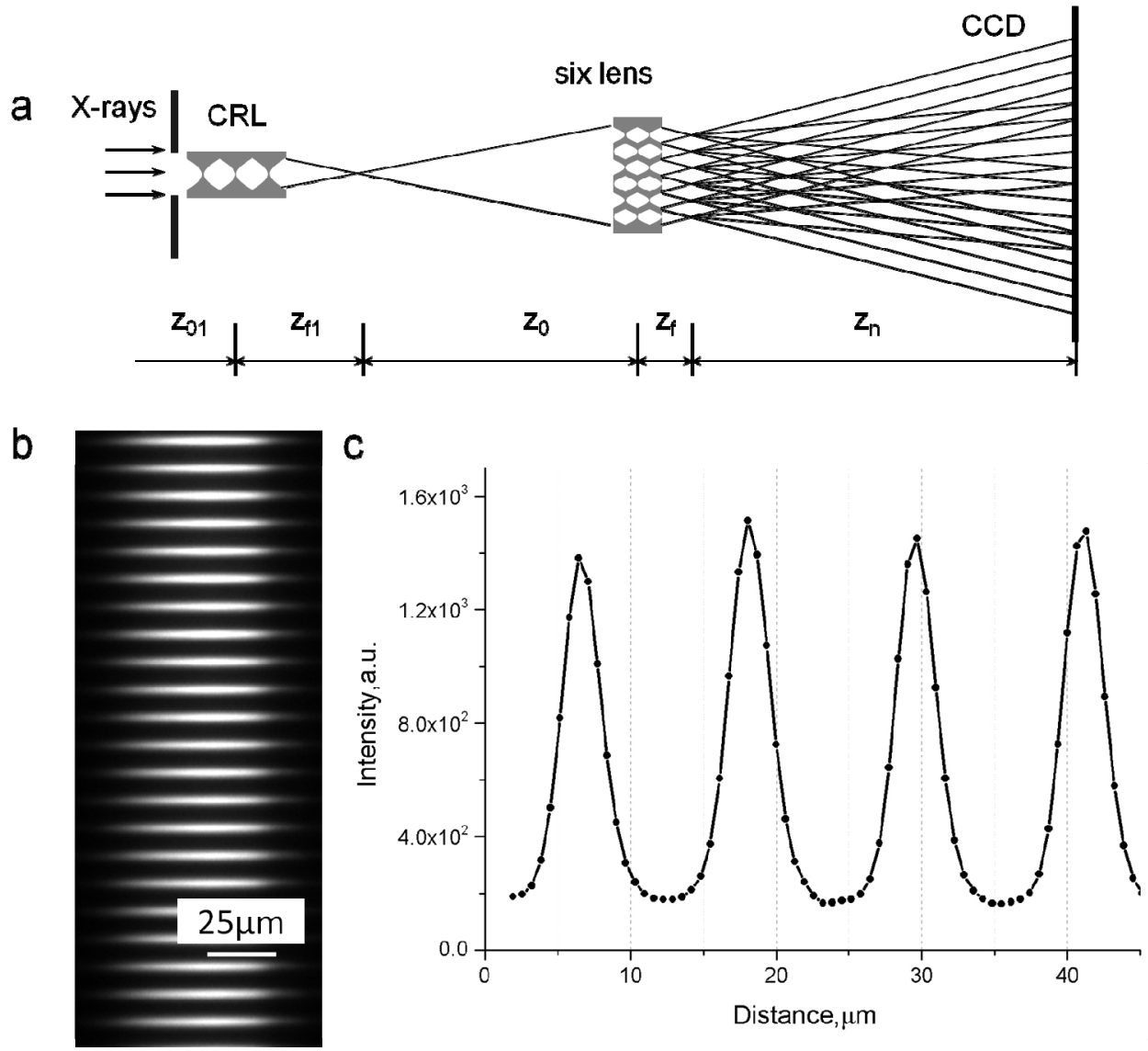

Fig. 4. (a) Experimental set-up fort the hexalens test with the point secondary source produced by a Si planar refractive lens. (b) An interference pattern generated by a hexalens, recorded at a $1 \AA$ wavelength. (c) The intensity variation obtained for the line though the centre of the fringe pattern.

\section{Conclusion}

A hexalens was designed and manufactured for hard X-rays. The lens arrays in the interferometer were arranged in a "chessboard" pattern such that the CRL arrays are shifted relative to each other by the distance equal to half length of the individual single lens. The new design allows us to overcome problems of the transmission of higher energies between lens arrays. Although all sets were designed for the X-ray energy range from 10 to $50 \mathrm{keV}$, in reality the energy diapason can be significantly extended from few $\mathrm{keV}$ up to $100 \mathrm{keV}$ by changing the nominal focal distance. The hexalens was studied at the energy range from 10 to $30 \mathrm{keV}$. The interference fringe images were recorded at different fractional Talbot distances. Narrowing of the interference fringe width produced by hexalens was confirmed experimentally through comparison with a bilens system. The enhancement of the fringe contrast was observed as well. The depth of field was studied and it is shown that it changed with the fraction of Talbot distance. Even at fractional 1/40 Talbot distance, the depth of field for the hexalens is more than 10 times greater compared to a Si nanolens with the same focal spot dimension. More than $90 \%$ interference fringe contrast was produced by the multilens interferometer under point source illumination. This gives us a hope that the hexalens interferometer structures are very good quality and do not exhibit a diffuse scattering at all. 


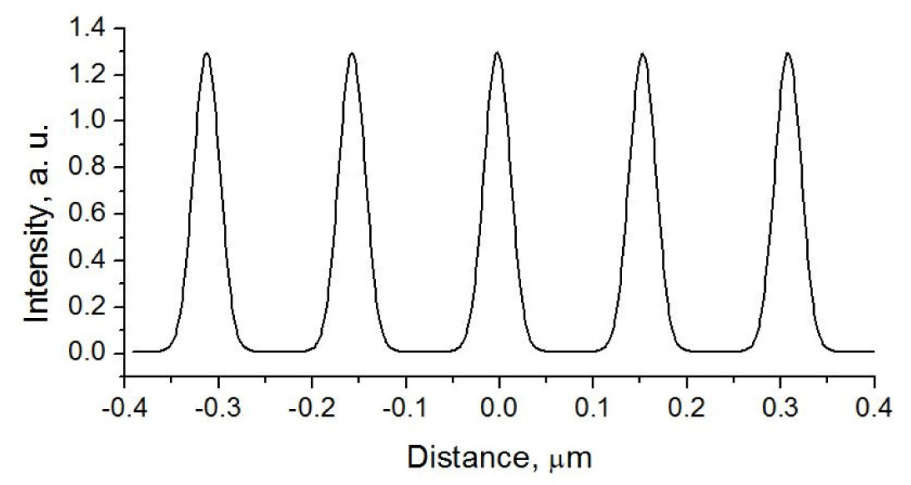

Fig. 5. Calculated interference pattern generated by sixlens interferometer for $10 \mu \mathrm{m}$ source size at $30 \mathrm{keV} \mathrm{X}$-rays.

For efficient use of the proposed sixlens interferometer it makes sense to address the question of the minimum period and fringe width of the interference pattern. Using the computer program developed here let us calculate the fringe pattern recorded at $z_{n}=12 \mathrm{~cm}$ fractional Talbot distance. The calculations were performed for $30 \mathrm{keV}$ X-rays and the source size was chosen 10 microns. The resulting interference pattern is depicted in Fig. 5. The periodicity of the pattern is $150 \mathrm{~nm}$, fringe width (FWHM) is $30 \mathrm{~nm}$, and visibility is close to $100 \%$.

This simple way to create an X-ray standing wave in paraxial geometry opens up the opportunity to develop new X-ray interferometry techniques to study natural and advanced man-made nanoscale materials, such as self-organised biosystems, photonic and colloidal crystals, and nanoelectronics materials. As a classical interferometer it can be used for phase contrast imaging and radiography. Finally it can be useful for the coherence characterization of the X-rays sources and free electron lasers. It should be noted that the proposed interferometer can be applied for electrochemical X-ray photolithography resulting in a direct non-contact pattern transfer onto an electrodeposited metal film [27]. Nanometer scale interference fringe pattern in combination with the short lifetime of radiolysis products and with a small spur radii and electron inelastic mean free paths in condensed matter will provide a possible means to rapidly improve the ultimate resolution of the proposed method to tens of nanometres (Talbot assisted lithography).

The same technology can be easily transferred to produce a multilens interferometer with an aperture up to $1 \mathrm{~mm}$ that is comparable with the beam size at the ESRF undulator beamlines.

\section{Acknowledgments}

The authors are very grateful to C. Detlefs for his help and support during the experiments at ID 06 beamline. The authors thank H. Simons for the helpful discussions. The work was supported by the Ministry of Science and Education of Russian Federation (grants $\mathrm{N}^{\circ}$ 14.Y26.31.0002, $\mathrm{N}^{\mathrm{o}}$ 02.G25.31.0086, and project 8364) and RFBR grant $\mathrm{N}^{\mathrm{o}} 13-02-00469$. 